\title{
Prestigio y estigmatización de 60 nombres propios en 44 sujetos pertenecientes a un centro de capacitación de adultos en situación de vulnerabilidad social (3)
}

\author{
Gastón Salamanca Gutiérrez², Macarena Millán Contreras³ ${ }^{3}$ Claudia \\ Rodríguez Gutiérrez ${ }^{4}$
}

\begin{abstract}
RESUMEN
Este artículo presenta el grado de prestigio/estigmatización de 60 nombres propios en 44 sujetos -22 hombres y 22 mujeres- en situación de vulnerabilidad social. El trabajo se focaliza en un rango etario que oscila entre los 22 y 46 años, en el caso de los hombres, y entre los 2I y 36 años, en el caso de las mujeres. Para la recopilación de datos, se aplica una encuesta que originalmente tenía 5 secciones, de las cuales, por razones que se detallan en el corpus de este estudio, solo dos fueron consideradas (evaluación de los nombres de acuerdo con su popularidad/impopularidad y vinculación con el nivel social alto o bajo). Respecto de los resultados obtenidos, se propone la existencia de una conciencia de norma sociolingüística menos prominente que en sujetos con un mayor nivel sociocultural y una cohesión mayor de las respuestas en el caso de los sujetos de sexo femenino.
\end{abstract}

Palabras clave: Sociolingüística, Sociología del Lenguaje, español de Chile, prestigio/ estigmatización, conciencia de norma.

Prestige and stigma of 60 names in 44 subjects belonging to a Training Center for adults in social vulnerability situation (3)

\section{ABSTRACT}

This article presents the level of prestige/stigma of 60 names in 44 subjects - 22 men and 22 women in situation of social vulnerability. The work focuses on an age range between 22 and 46 years in the case of men and 2I and 36 years in the case of women. For data collection, the study applied a survey, which originally had five sections, but, for reasons detailed in the body of this study, it considers only two of them (evaluation of names according to their popularity/unpopularity and their linkage to high or low social level). Regarding the results, it found a kind of lack of awareness of sociolinguistic rules

Este artículo constituye la tercera parte del estudio denominado "Estereotipos sociales asociados a seis nombres propios en una muestra de cuarenta sujetos de nivel educacional superior", el cual fue publicado en Foro Educacional $N^{\circ} 23$ y No 24.

2 Gastón Salamanca es Doctor en Lingüistica, académico de la Facultad de Humanidades y Arte de la Universidad de Concepción. E-mail: gaston.salamanca@gmail.com

3 Macarena Millán Contreras es Licenciada en Educación con Mención en Español por la Universidad de Concepción. E-mail: mmillan.contreras@gmail.com

4 Claudia Rodríguez Gutiérrez es Licenciada en Educación con Mención en Español por la Universidad de Concepción. E-mail: clau.rodriguez.gutierrez@gmail.com 
in subjects with a lower socio-cultural level and greater cohesion of responses in the case of female subjects.

Key words: Sociolinguistics, Sociology of Language, spanish, Chile, prestige/stigma, awareness of norms.

\section{Introducción}

De acuerdo con Valdivieso (I983, p. 137), la valoración social de la lengua "implica un ordenamiento jerárquico de las variaciones: existen usos que tienen prestigio y usos que son estigmatizados”. El español de Chile no está exento de dicho fenómeno, lo que queda demostrado mediante algunos estudios de especialistas del área en los distintos niveles de la lengua. Por ejemplo, en el plano morfosintáctico, es claro que una construcción como "le voy a decirle", donde se duplica el clítico en función de objeto indirecto, es estigmatizada. En el plano léxico, también, se observa esta situación. Así, por ejemplo, es claro que palabras como despailarse, dentren, baiga, se asocian con un estrato social deprivado.

Ahora, si se piensa en el abordaje de este fenómeno desde la Lingüística en Chile, parece ser que el nivel fonético-fonológico es el que ha recibido una mayor atención. Un ejemplo paradigmático de ello es el estudio de la variación del fonema / $\hat{c} /\left(/ \mathrm{t} \int /\right)$. En efecto, Valdivieso (1983) y Tassara (1992) establecen claramente que la variante fricativa š $\left(\left[\int\right]\right)$ de este fonema posee una fuerte estigmatización y que la variante africada es prestigiosa (o, al menos, no marcada). Recientemente, Figueroa, Salamanca y Nanculeo (2013) han demostrado que la coordenada sociolingüística prestigio/ estigmatización se puede aplicar no sólo a esta variable, sino que a toda una clase constituida por el grupo fónico / tr/, el fonema vibrante múltiple /r/ y el fonema africado /d $/$.

Esta coordenada sociolingüística (prestigio/estigmatización), relevada por Valdivieso en el ámbito fonético y aplicada en trabajos como los de Salamanca y Valverde (2009), Figueroa, Soto y Nanculeo (2010), Salamanca (2010) y Salamanca y Pereira (2013), orienta, también, la realización de este artículo. Así, su objetivo general es determinar, dentro de un conjunto de nombres propios, cuáles son 
considerados más prestigiosos y cuáles más estigmatizados por un grupo de sujetos pertenecientes a un centro de capacitación de adultos, cuyo foco son personas en situación de vulnerabilidad social. De forma más específica, se pretende establecer el grado de popularidad/desprestigio que los sujetos atribuyen a 60 nombres propios y la relación que establecen entre estos y los estratos sociales alto y bajo. La manera en que se lleva a cabo la investigación es a través de una encuesta que posee 60 nombres propios (30 femeninos y 30 masculinos), los que, explícita o implícitamente, deben ser clasificados por los encuestados, guiándose para ello por tres criterios básicos propuestos por los investigadores: grado de popularidad, asociación a nivel social y grado de preferencia o rechazo personal.

Como se aprecia, el presente estudio se inscribe dentro de la Sociolingüística y la Sociología del Lenguaje, pues apunta al estudio "del hecho lingüístico en toda su dimensión social" (Silva-Corvalán, 200I, p. 2); a la vez, que se centra en el estudio de la "interacción entre el uso de una lengua y la organización social del comportamiento humano" (Fishman 1972, en Silva-Corvalán, 200I, p. 4); en este caso, se refiere a una sociedad altamente estratificada que segmenta a sus individuos, entre otros criterios, por sus nombres propios.

\section{Marco de referencia}

\subsection{Sociolingüística}

La Sociolingüística, según lo señalado por Silva-Corvalán (200I), se define como la disciplina que estudia la relación que se establece entre fenómenos lingüísticos y factores sociales, que pueden ser: factores individuales (como edad, raza, sexo y nivel de instrucción); la situación de enunciación, es decir, el contexto en que acontecen los hechos lingüísticos; los diversos sistemas de organización propios de una sociedad (político, económico, social y geográfico), y, por último, los aspectos históricos y étnico-culturales.

De la descripción presentada, se desprende que el objeto de estudio de la Sociolingüística es "el hecho lingüístico en toda su dimensión 
social” (Silva-Corvalán, 200I, p. 2), priorizando la función comunicativa y social propia de toda lengua, centrándose en sus variadas formas de uso y considerándola un objeto complejo en el que se articulan reglas lingüísticas y sociales.

En Sociolingüística se ha propuesto que la lengua tiene un valor simbólico (Cooper, 1989; Silva-Corvalán, 200I; Carbajal, 2006; Pujol, 2013) en el sentido de que utilizar un dialecto simboliza la pertenencia de un individuo a cierto grupo social; así, por ejemplo, en el área de la educación existen algunas posturas que privilegian la enseñanza del dialecto estándar, pues consideran que pronunciar el español estándar es reflejo de superioridad social.

\subsection{Actitudes lingüísticas}

La Sociolingüística se ocupa de un tipo específico de actitudes, esto es, las de tipo lingüístico, que pueden definirse como aquellas que tratan acerca de las actitudes hacia la lengua e incluso pueden ampliarse y "abarcar las actitudes hacia los hablantes de una lengua o dialecto particulares” (Fasold, I996, p. 23I) o, más aún, incluir actitudes respecto de la conservación y proyectos de planificación lingüística. Además, es posible suponer un vínculo entre las actitudes y ciertos fenómenos sociales. Por ejemplo, Wolff (I959, en Fasold, I996) postula que las actitudes lingüísticas pueden influir en la inteligibilidad de una variedad lingüística, esto es, si se consideran dos variedades lingüísticas que posean un vínculo estrecho, cabe la posibilidad de que los sujetos que utilicen la variedad que posea un estatus más alto no comprendan a su contraparte, pero la variedad de estatus inferior sí entenderá a aquella.

De forma general, considerando lo señalado por Lastra, "las actitudes reflejan la estratificación de la sociedad, e incluso los miembros de las clases bajas o de otras minorías comparten muchas veces las actitudes de los grupos dominantes" (Lastra, I992, p. 419).

\subsection{Prestigio y estigmatización}

El prestigio lingüístico, se refiere al 
Valor positivo que ciertas variables lingüísticas tienen en cuanto a facilitar el ascenso en la escala social y también al valor que tienen las formas lingüísticas estándares, reconocidas y aceptadas por las gramáticas normativas y generalmente asociadas con la clase media alta culta. (Silva-Corvalán, 2001, p. 99)

Los patrones de evaluación que sirven para determinar lo que es o no prestigioso son, de acuerdo a estudios sociolingüísticos, diferentes si se hace referencia al habla femenina o masculina, pues lo que para un género es prestigioso no lo es, necesariamente, para el otro.

A pesar de que los sujetos están conscientes de lo que es prestigioso, en muchos casos continúan utilizando variables lingüísticas de bajo prestigio, lo que se debe, según lo señalado por Silva-Corvalán, a la presión social respecto de la identificación con un grupo (lo que al parecer se manifiesta mayormente en los hombres). Dicha conducta se relaciona con el fenómeno de "prestigio encubierto", que se evidencia en el modo en que se evalúa de forma subjetiva el habla de otros y la propia.

\subsection{Antecedente para esta investigación}

El estudio de Salamanca y Pereira (2013) se relaciona directamente con nuestra investigación, puesto que determina el grado de prestigio y estigmatización de 60 nombres propios en 40 sujetos de nivel educacional superior. Las principales conclusiones a las que llegan estos investigadores son las siguientes:

- Existe una alta conciencia de norma sociolingüística por parte de los sujetos encuestados, lo que se refleja en la alta concentración de las preferencias y la alta cantidad de votos recibida por algunos nombres, especialmente en las preguntas relacionadas con estigmatización.

- Las votaciones de las mujeres son las que concuerdan en mayor medida con las expectativas otorgadas en el diseño del instrumento.

- La cantidad de nombres votados por los encuestados y encuestadas es siempre inferior en las preguntas relativas a estigmatización, lo que revela que la elección de un nombre hace 
emerger en estos sujetos las distintas asunciones culturales que han internalizado en su comunidad de habla.

\section{Metodología}

\subsection{Sujetos}

La muestra está compuesta por 44 estudiantes: 22 hombres, cuyas edades oscilan entre los 22 y 46 años; y 22 mujeres, cuyas edades fluctúan entre los 2I y 36 años. Los sujetos son alumnos de la institución educativa INFOCAP (Instituto de Formación y Capacitación Popular), pertenecientes a tres oficios: Especialistas en Belleza, Gastronomía e Instalaciones Eléctricas. Dicho centro de capacitación acoge mayoritariamente a adultos que, por motivos principalmente socioeconómicos, no han podido acceder a estudios superiores y, por ende, a una profesión, lo que se correlaciona con el hecho de que pertenecen a un sector socioeconómico y cultural predominantemente bajo. El motivo por el cual se decidió aplicar el instrumento en un grupo de sujetos pertenecientes a un nivel social bajo fue para constatar si la selección de nombres que realizan muestra tendencias sistemáticas y, de ser así, si se presentan en la misma medida en que ocurren en sujetos de nivel educacional superior y estrato medio/medio alto, de acuerdo con el reporte de Salamanca y Pereira (2013).

\subsection{Instrumento}

El instrumento utilizado para recopilar la información es la encuesta que se presenta en Salamanca y Pereira (2013), en que se presentan 60 nombres propios ( 30 masculinos y 30 femeninos), de los cuales I0 tenían la expectativa de ser considerados como prestigiosos, I0 sin una asociación clara con prestigio o estigmatización, y I0 como estigmatizados.

El total de encuestas aplicadas cuyos nombres propios eran femeninos fue de 36, I7 pertenecían a estudiantes de Especialista en Belleza, I8 a Gastronomía y I al oficio de Instalaciones Eléctricas. De este total, se seleccionaron 22 , según criterios relacionados con la edad y de 
acuerdo al grado de completación de la encuesta; II correspondían a los estudiantes de Especialista en Belleza y II a Gastronomía.

El total de encuestas aplicadas cuyos nombres propios eran masculinos fue de 27; 26 de ellas pertenecían a alumnos de Instalaciones Eléctricas y I a Gastronomía. Fue necesario seleccionar las encuestas más idóneas, de acuerdo con los parámetros ya mencionados y se obtuvo un total de 22, que correspondían solo a los estudiantes de Instalaciones Eléctricas, nivelándose de esta forma con la cantidad de encuestas cuyos nombres propios eran femeninos.

Debido a la extensión de la encuesta y a que los ítems 4 y 5 no fueron respondidos por un número importante de sujetos, para el análisis de datos solo se consideraron las preguntas I.a, 2.a y 3.a. ${ }^{\text {I }}$

\subsection{Nombres seleccionados}

A continuación, se presentan los 60 nombres propios evaluados por los sujetos, divididos, de acuerdo con las expectativas iniciales de los investigadores, en las categorías estigmatizado, prestigioso y neutro.

\subsubsection{Nombres masculinos}

\begin{tabular}{|l|l|l|}
\hline \multicolumn{1}{|c|}{ ESTIGMATIZADOS } & \multicolumn{1}{c|}{ PRESTIGIOSOS } & \multicolumn{1}{c|}{ NEUTROS } \\
\hline Brian & I. Cristóbal & I. Rodrigo \\
\hline Michael & 2. Sebastián & 2. Marcelo \\
\hline Jason & 3. Martín & 3. Daniel \\
\hline Jeremy & 4. Alonso & 4. Andrés \\
\hline Byron & 5. Tomás & 5. Claudio \\
\hline Harry & 6. Ignacio & 6. Alejandro \\
\hline Malcom & 7. Joaquín & 7. Pablo \\
\hline Jordan & 8. Agustín & 8. Héctor \\
\hline Kevin & 9. León & 9. Mauricio \\
\hline Justin & I0. Máximo & I0. Felipe \\
\hline
\end{tabular}

Por razones de espacio, los resultados obtenidos en la pregunta 3.a no serán incluidos en este artículo; no obstante, pueden encontrarse en la Tesis para optar al Grado de Licenciado en Educación con Mención en Español de Millán y Rodríguez (2012). 


\subsubsection{Nombres femeninos}

\begin{tabular}{|l|l|l|}
\hline \multicolumn{1}{|c|}{ ESTIGMATIZADOS } & \multicolumn{1}{c|}{ PRESTIGIOSOS } & \multicolumn{1}{c|}{ NEUTROS } \\
\hline I. Joselyn & I. Martina & I. Carla \\
\hline 2. Melany & 2. Sofía & 2. Carolina \\
\hline 3. Maryori & 3. Isidora & 3. Andrea \\
\hline 4. Yaritza & 4. Antonia & 4. Claudia \\
\hline 5. Sharon & 5. Josefa & 5. Alejandra \\
\hline 6. Shirley & 6. Ignacia & 6. Daniela \\
\hline 7. Nayareth & 7. María Paz & 7. Cecilia \\
\hline 8. Yamileth & 8. Agustina & 8. Paulina \\
\hline 9. Melody & 9. Julieta & 9. Fabiola \\
\hline I0. Britney & I0. Matilde & 10. Natalia \\
\hline
\end{tabular}

\subsubsection{Orden de los nombres dentro de la encuesta}

\subsubsection{Nombres masculinos}

\begin{tabular}{|l|l|l|l|l|l|}
\hline Cristóbal & Máximo & Jason & Héctor & Tomás & Jordan \\
\hline Alonso & Jeremy & Andrés & Rodrigo & Kevin & Joaquín \\
\hline Malcom & Alejandro & Michael & Justin & Agustín & Ignacio \\
\hline Pablo & León & Claudio & Mauricio & Brian & Sebastián \\
\hline Daniel & Byron & Harry & Felipe & Marcelo & Martín \\
\hline
\end{tabular}

\subsubsection{Nombres femeninos}

\begin{tabular}{|l|l|l|l|l|l|}
\hline Alejandra & Sharon & Fabiola & Ignacia & Martina & Claudia \\
\hline Isidora & Joselyn & $\mathrm{M}^{\text {a }}$ Paz & Carolina & Andrea & Melany \\
\hline Shirley & Sofía & Nayareth & Maryori & Cecilia & Carla \\
\hline Antonia & Agustina & Yamileth & Daniela & Britney & Julieta \\
\hline Josefa & Paulina & Matilde & Yaritza & Natalia & Melody \\
\hline
\end{tabular}

\section{Análisis y resultados}

\subsection{Consideraciones previas}

Como se ha señalado, la lista de nombres se elaboró inicialmente, considerando la siguiente expectativa: I0 nombres serían asociados con prestigio; I0 no presentarían una asociación prominente y I0 serían asociados con estigmatización. Esta expectativa inicial se 
modificó levemente en su versión final, en el siguiente sentido: a dos nombres masculinos (León y Máximo) y dos nombres femeninos (Julieta y Matilde) se le asignó más de una expectativa (positiva y neutra). De este modo, entonces, los nombres fueron calificados de cuatro formas distintas, lo que se expresa mediante la siguiente simbología:

$+\quad$ : expectativa de valoración positiva del nombre (nombre prestigioso)

+- : expectativa de valoración neutra del nombre (sin asociación con prestigio/estigmatización)

- $\quad$ : expectativa de valoración negativa del nombre (nombre estigmatizado).

+/+- : expectativa de valoración positiva y neutra.

\subsection{Resultados versión sexo femenino}

3.2.1. En relación con la pregunta "¿Con cuál de los siguientes nombres crees que tus amigos se reirían de ti?"

\begin{tabular}{|c|c|c|c|c|}
\hline $\mathrm{N}^{\circ}$ & NOMBRE & $\mathrm{N}$ & PORCENTAJE & EXPECTATIVA \\
\hline I & Shirley & $2 I$ & $95 \%$ & - \\
\hline 2 & Britney & 17 & $77 \%$ & - \\
\hline 3 & Sharon & $\mathrm{I} 4$ & $64 \%$ & - \\
\hline 4 & Melody & $\mathrm{I} 3$ & $59 \%$ & - \\
\hline 5 & Yamileth & 13 & $59 \%$ & - \\
\hline 6 & Maryori & 9 & $41 \%$ & - \\
\hline 7 & Agustina & 8 & $36 \%$ & + \\
\hline 8 & Yaritza & 8 & $36 \%$ & - \\
\hline 9 & Julieta & 7 & $32 \%$ & $+/+-$ \\
\hline 10 & Matilde & 5 & $23 \%$ & $+/+-$ \\
\hline II & Nayareth & 4 & $18 \%$ & - \\
\hline $\mathrm{I} 2$ & Isidora & 3 & $\mathrm{I} 4 \%$ & + \\
\hline I3 & Ignacia & 2 & $9 \%$ & + \\
\hline $\mathrm{I} 4$ & Martina & 2 & $9 \%$ & + \\
\hline I5 & Melany & 2 & $9 \%$ & - \\
\hline 16 & Natalia & 2 & $9 \%$ & +- \\
\hline 17 & Joselyn & I & $5 \%$ & - \\
\hline 18 & María Paz & I & $5 \%$ & + \\
\hline
\end{tabular}




\section{Observaciones}

Inicialmente, la expectativa se cumple, pues los nombres cuya perspectiva de valoración era claramente negativa se sitúan en las seis primeras posiciones; sin embargo, con un $36 \%$ de preferencias, sorprende que en el número siete se ubique el nombre Agustina, puesto que su expectativa de valoración era claramente positiva.

A continuación, se encuentran los nombres Julieta y Matilde, con un $32 \%$ y $23 \%$ de preferencias, respectivamente, lo cual también es destacable dado que no se aviene con las expectativas positiva y neutra que se tenía respecto de ellos. Más sorprendente es aún si se considera que tienen mayor votación que un nombre como Nayaretb. Posteriormente, destaca Isidora, pues obtuvo un I $4 \%$ de preferencias, superando en este estatus a nombres como Melany y Joselyn.

Al establecer una comparación con los resultados obtenidos por Salamanca y Pereira (2013), es posible señalar que existe una cohesión mucho mayor en las votaciones emitidas por los sujetos con un nivel educacional superior, pues en ese caso los nombres considerados fueron solo 9, mientras que en el presente estudio la cifra asciende a I8.

Otro aspecto importante es que en ambos casos Shirley se sitúa en la primera posición, recibiendo un $95 \%$ de los votos.

Finalmente, es digno de mención que en ambos estudios el nombre Sharon ocupa el tercer lugar dentro de las votaciones; no obstante, en el caso de los sujetos con nivel educacional superior alcanza un 90,47\% (I9 menciones), frente al 64\% (I4 menciones) otorgado por los sujetos adultos en situación de vulnerabilidad social.

\subsubsection{En relación con la pregunta “¿Con cuál de los siguientes nombres crees que serías más popular entre tus amigos?"}

\begin{tabular}{|l|l|l|l|l|}
\hline $\mathrm{N}^{\circ}$ & \multicolumn{1}{|c|}{ NOMBRE } & \multicolumn{1}{|c|}{$\mathrm{N}$} & PORCENTAJE & EXPECTATIVA \\
\hline I & Ignacia & I7 & $77 \%$ & + \\
\hline 2 & Martina & I6 & $73 \%$ & + \\
\hline 3 & Antonia & I4 & $64 \%$ & + \\
\hline 4 & María Paz & I3 & $59 \%$ & + \\
\hline 5 & Alejandra & II & $50 \%$ & +- \\
\hline
\end{tabular}




\begin{tabular}{|l|l|l|l|l|}
\hline 6 & Josefa & 9 & $41 \%$ & + \\
\hline 7 & Sofía & 8 & $36 \%$ & + \\
\hline 8 & Carolina & 7 & $32 \%$ & +- \\
\hline 9 & Isidora & 7 & $32 \%$ & + \\
\hline I0 & Daniela & 6 & $27 \%$ & +- \\
\hline II & Agustina & 3 & $\mathrm{I} \%$ & + \\
\hline I2 & Andrea & 3 & $\mathrm{I} \% \%$ & +- \\
\hline I3 & Carla & 3 & $\mathrm{I} \% \%$ & +- \\
\hline I4 & Claudia & 3 & $\mathrm{I} \%$ & +- \\
\hline I5 & Fabiola & 3 & $\mathrm{I} \%$ & +- \\
\hline I6 & Joselyn & 3 & $\mathrm{I} \%$ & - \\
\hline I7 & Julieta & 2 & $9 \%$ & $+/+-$ \\
\hline I8 & Matilde & 2 & $9 \%$ & $+/+-$ \\
\hline I9 & Melany & 2 & $9 \%$ & - \\
\hline 20 & Melody & 2 & $9 \%$ & - \\
\hline 21 & Paulina & 2 & $9 \%$ & +- \\
\hline 22 & Britney & I & $5 \%$ & - \\
\hline 23 & Cecilia & I & $5 \%$ & +- \\
\hline 24 & Natalia & I & $5 \%$ & +- \\
\hline 25 & Nayareth & I & $5 \%$ & - \\
\hline & & & & \\
\hline
\end{tabular}

\section{Observaciones}

A partir de la tabla expuesta, se observa cómo con los cuatro primeros nombres se cumplen las expectativas. Sin embargo, en la posición número cinco se encuentra el nombre Alejandra (con un $50 \%$ de preferencias), lo cual sorprende, pues no estaba dentro de las expectativas que este nombre se ubicara en un lugar prominente dentro de los nombres prestigiosos (superando, incluso, a Josefa, Sofía e Isidora). Era, de hecho, considerado uno de los nombres "neutros".

En esta misma dirección, aunque con un porcentaje menor de preferencias (32\%), llama la atención que Carolina comparta el mismo porcentaje que Isidora y supere a Agustina. Una situación similar ocurre con el nombre Daniela, con respecto a Agustina.

Con un I4\% de preferencias, destaca la aparición del nombre Joselyn, puesto que respecto de él se tenía una expectativa de valoración 
claramente negativa y, por lo tanto, no se preveía que tuviera más votación que Matilde, por ejemplo.

Al establecer una comparación con los resultados obtenidos por Salamanca y Pereira (2013), es posible señalar que existe una mayor cohesión en las votaciones emitidas por los sujetos con un nivel educacional superior, pues en ese caso los nombres considerados fueron 20, mientras que en el presente estudio la cifra asciende a 25.

Por otra parte, destaca el porcentaje de votos obtenido por Agustina, el cual alcanza un $66,66 \%$ en sujetos con nivel educacional superior, frente al I4\% en los sujetos en situación de vulnerabilidad social.

Otra diferencia entre ambos estudios radica en que, en el caso de los sujetos con nivel educacional superior, la expectativa se cumple en forma consecutiva en los primeros 9 nombres, mientras que en los sujetos adultos en situación de vulnerabilidad social, esto ocurre solo en los cuatro primeros.

\subsubsection{En relación con la pregunta "¿Cuáles de estos nombres} relacionas con un nivel social bajo?"

\begin{tabular}{|l|c|c|c|c|}
\hline$N^{\circ}$ & NOMBRE & N & PORCENTAJE & EXPECTATIVA \\
\hline I & Shirley & I4 & $64 \%$ & - \\
\hline 2 & Britney & I2 & $55 \%$ & - \\
\hline 3 & Yamileth & I2 & $55 \%$ & - \\
\hline 4 & Sharon & II & $50 \%$ & - \\
\hline 5 & Yaritza & II & $50 \%$ & - \\
\hline 6 & Melody & I0 & $45 \%$ & - \\
\hline 7 & Maryori & 7 & $32 \%$ & +- \\
\hline 8 & Claudia & 6 & $27 \%$ & +- \\
\hline 9 & Nayareth & 6 & $27 \%$ & - \\
\hline I0 & Carla & 5 & $23 \%$ & +- \\
\hline II & Melany & 5 & $23 \%$ & +- \\
\hline I2 & Andrea & 4 & I8\% & +- \\
\hline I3 & Alejandra & 3 & I4\% & + - - \\
\hline I4 & Carolina & 3 & I4\% & I4\% \\
\hline I5 & Matilde & 3 & & - \\
\hline
\end{tabular}




\begin{tabular}{|c|c|c|c|c|}
\hline I6 & Natalia & 3 & $\mathrm{I} \%$ & +- \\
\hline I7 & Agustina & 2 & $9 \%$ & + \\
\hline I8 & Cecilia & 2 & $9 \%$ & +- \\
\hline I9 & Daniela & 2 & $9 \%$ & +- \\
\hline 20 & Fabiola & 2 & $9 \%$ & +- \\
\hline 21 & Antonia & I & $5 \%$ & + \\
\hline 22 & Isidora & I & $5 \%$ & + \\
\hline 23 & Joselyn & I & $5 \%$ & - \\
\hline 24 & María Paz & I & $5 \%$ & + \\
\hline 25 & Martina & I & $5 \%$ & + \\
\hline 26 & Paulina & I & $5 \%$ & +- \\
\hline 27 & Sof́́a & I & $5 \%$ & + \\
\hline
\end{tabular}

\section{Observaciones}

Inicialmente, la expectativa se cumple, puesto que los siete primeros nombres asociados con nivel bajo poseen una expectativa de valoración negativa. Luego destaca la aparición del nombre Claudia dado que se preveía con una valoración neutra, y, sin embargo, comparte el mismo porcentaje (27\%) que Nayareth, el cual sí se preveía asociado con un estrato bajo. Asimismo, destaca la aparición de los nombres Carla (23\%) y Andrea (I8\%), puesto que respecto de ellos no se preveía su asociación con un estrato determinado.

Destaca, también, la posición del nombre Joselyn, pues, con apenas un 5\% de votación, comparte la misma posición que Antonia, Isidora, María Paz, Martina y Sofía, los cuales se preveían sin menciones para el estrato bajo.

Al establecer una comparación con los resultados obtenidos por Salamanca y Pereira (20I3), es posible señalar que existe una cohesión mucho mayor en las votaciones emitidas por los sujetos con un nivel educacional superior, pues en ese caso los nombres considerados fueron solo II, mientras que en el presente estudio la cifra asciende a 27.

Otro dato interesante es la coincidencia del nombre Shirley, encabezando ambas tablas, aunque con porcentajes distintos: en el caso del estudio de Salamanca y Pereira (2013), alcanza un 90,47\%, mientras que en este estudio solamente alcanza un $64 \%$ de los votos. 
Por último, se observa que en el trabajo de Salamanca y Pereira (2013) los I0 nombres con expectativa de valoración negativa se sitúan en las I0 primeras posiciones, lo cual no ocurre en la presente investigación, donde, si bien 7 de los nombres con expectativa de valoración negativa encabezan la tabla, dos se intercalan con nombres neutros, y uno, Joselyn, rompe totalmente con la expectativa, pues solo obtiene un voto, alcanzando un 5\%.

\subsubsection{En relación con la pregunta "¿Cuáles de estos nombres relacionas con un nivel social alto?"}

\begin{tabular}{|l|c|c|c|c|}
\hline $\mathrm{N}^{\circ}$ & NOMBRE & $\mathrm{N}$ & PORCENTAJE & EXPECTATIVA \\
\hline $\mathrm{I}$ & Josefa & $\mathrm{I} 5$ & $68 \%$ & + \\
\hline 2 & Isidora & $\mathrm{I} 4$ & $64 \%$ & + \\
\hline 3 & María Paz & $\mathrm{I} 4$ & $64 \%$ & + \\
\hline 4 & Antonia & $\mathrm{I} 3$ & $59 \%$ & + \\
\hline 5 & Ignacia & $\mathrm{I} 3$ & $59 \%$ & + \\
\hline 6 & Sofia & $\mathrm{I} 2$ & $55 \%$ & + \\
\hline 7 & Martina & $\mathrm{I} 0$ & $45 \%$ & + \\
\hline 8 & Agustina & 4 & $18 \%$ & + \\
\hline 9 & Alejandra & 4 & $18 \%$ & +- \\
\hline I0 & Matilde & 4 & $18 \%$ & $+/+-$ \\
\hline II & Andrea & 3 & $14 \%$ & +- \\
\hline I2 & Carla & 3 & $14 \%$ & +- \\
\hline I3 & Julieta & 3 & $14 \%$ & $+/+-$ \\
\hline I4 & Sharon & 3 & $14 \%$ & - \\
\hline I5 & Carolina & 2 & $9 \%$ & +- \\
\hline I6 & Claudia & 2 & $9 \%$ & +- \\
\hline I7 & Daniela & 2 & $9 \%$ & +- \\
\hline I8 & Melany & 2 & $9 \%$ & - \\
\hline I9 & Britney & I & $5 \%$ & - \\
\hline 20 & Cecilia & I & $5 \%$ & +- \\
\hline 21 & Fabiola & I & $5 \%$ & +- \\
\hline 22 & Joselyn & I & $5 \%$ & - \\
\hline 23 & Melody & I & $5 \%$ & - \\
\hline 24 & Paulina & I & $5 \%$ & +- \\
\hline 25 & Yamileth & I & $5 \%$ & - \\
\hline & & & & \\
\hline
\end{tabular}




\section{Observaciones}

En primer lugar, se visualiza que las expectativas son alcanzadas en los ocho primeros nombres. Luego, destaca el caso de Alejandra, el cual no se preveía asociado con un estrato sociocultural determinado; sin embargo, recibe la misma votación que los nombres Agustina y Matilde (I8\%), los cuales sí se preveían vinculados con el estrato alto.

Con un I4\% de preferencias, destaca, asimismo, el nombre Sharon, pues se preveía asociado con un estrato bajo.

Al establecer una comparación con los resultados obtenidos por Salamanca y Pereira (2013), también, es posible señalar que existe una cohesión mucho mayor en las votaciones emitidas por los sujetos con un nivel educacional superior, pues en ese caso los nombres considerados fueron solo I5, mientras que en el presente estudio la cifra asciende a 25 .

Otro aspecto destacable a la hora de comparar ambos estudios es que los sujetos de nivel educacional superior otorgaron un 90,47\% de las votaciones al nombre Agustina, porcentaje que decae a un I8\% en los datos de la presente investigación.

Se destaca, además, que los sujetos en situación de vulnerabilidad social sitúan el nombre Josefa en la primera posición con un $68 \%$ de los votos (I5 menciones), mientras que los sujetos con nivel educacional superior lo posicionan en séptimo lugar con un 47,6I\% de las preferencias (I0 menciones).

\subsection{Resultados versión sexo masculino}

\subsubsection{En relación con la pregunta “¿Con cuál de los siguientes nombres crees que tus amigos se reirían de ti?"}

\begin{tabular}{|l|c|c|c|c|}
\hline $\mathrm{N}^{\circ}$ & NOMBRE & $\mathrm{N}$ & PORCENTAJE & EXPECTATIVA \\
\hline $\mathrm{I}$ & León & I5 & $68 \%$ & $+/+-$ \\
\hline 2 & Harry & I3 & $59 \%$ & - \\
\hline 3 & Byron & II & $50 \%$ & - \\
\hline 4 & Justin & II & $50 \%$ & - \\
\hline 5 & Malcom & II & $50 \%$ & - \\
\hline
\end{tabular}




\begin{tabular}{|c|c|c|c|c|}
\hline 6 & Jeremy & 10 & $45 \%$ & - \\
\hline 7 & Jordan & 9 & $41 \%$ & - \\
\hline 8 & Jason & 6 & $27 \%$ & - \\
\hline 9 & Kevin & 5 & $23 \%$ & - \\
\hline 10 & Michael & 5 & $23 \%$ & - \\
\hline II & Rodrigo & 5 & $23 \%$ & + \\
\hline 12 & Tomás & 5 & $23 \%$ & + \\
\hline 13 & Agustín & 3 & $\mathrm{I} 4 \%$ & + \\
\hline I4 & Andrés & 3 & $\mathrm{I} 4 \%$ & +- \\
\hline 15 & Brian & 3 & $14 \%$ & - \\
\hline 16 & Joaquín & 3 & $\mathrm{I} 4 \%$ & + \\
\hline 17 & Máximo & 3 & $14 \%$ & $+/+$ \\
\hline 18 & Alonso & 2 & $9 \%$ & + \\
\hline 19 & Cristóbal & 2 & $9 \%$ & + \\
\hline 20 & Héctor & 2 & $9 \%$ & +- \\
\hline $2 \mathrm{I}$ & Martín & 2 & $9 \%$ & + \\
\hline 22 & Claudio & $\mathrm{I}$ & $5 \%$ & + \\
\hline 23 & Felipe & $\mathrm{I}$ & $5 \%$ & + \\
\hline 24 & Marcelo & I & $5 \%$ & +- \\
\hline
\end{tabular}

\section{Observaciones}

Es interesante que en el primer lugar aparezca el nombre León (68\%), pues respecto de él se tenía una expectativa de valoración que fluctuaba entre positiva y neutra. Los nombres que siguen cumplen plenamente las expectativas, con porcentajes que varían entre el 59\% y el $23 \%$.

Con un $23 \%$ de los votos destaca la presencia del nombre Tomás, pues respecto de este se tenía la expectativa de ser asociado con popularidad. Algo similar sucede con los nombres Agustín y Joaquín, respecto de los cuales también se tenía la expectativa de ser asociados con popularidad.

Al establecer una comparación con los resultados obtenidos por Salamanca y Pereira (2013), se aprecia, nuevamente, que existe una cohesión mucho mayor en las votaciones emitidas por los sujetos con un nivel educacional superior, pues en ese caso los nombres 
considerados fueron solo I6, mientras que en el presente estudio la cifra asciende a 24 .

Al comparar ambos estudios, destaca, además, que los sujetos masculinos de nivel educacional superior cumplen plenamente las expectativas dado que distribuyen los 10 nombres con expectativa de valoración negativa en las primeras posiciones de la tabla, mientras que en el presente estudio la tabla la encabeza el nombre León, respecto del cual no se tenía una expectativa de asociación con un estrato bajo.

Por último, destaca la baja votación (I4\%) del nombre Brian, pues en los sujetos de nivel educacional superior alcanza un $84,25 \%$.

\subsubsection{En relación con la pregunta "¿Con cuál de los siguientes} nombres crees que serías más popular entre tus amigos?"

\begin{tabular}{|l|c|c|c|c|}
\hline $\mathrm{N}^{\circ}$ & NOMBRE & $\mathrm{N}$ & PORCENTAJE & EXPECTATIVA \\
\hline $\mathrm{I}$ & Sebastián & $\mathrm{I} 2$ & $55 \%$ & + \\
\hline 2 & Cristóbal & 8 & $36 \%$ & + \\
\hline 3 & Daniel & 8 & $36 \%$ & +- \\
\hline 4 & Ignacio & 8 & $36 \%$ & + \\
\hline 5 & Máximo & 8 & $36 \%$ & $+/+$ \\
\hline 6 & Brian & 7 & $32 \%$ & - \\
\hline 7 & Andrés & 6 & $27 \%$ & +- \\
\hline 8 & Héctor & 6 & $27 \%$ & +- \\
\hline 9 & Mauricio & 6 & $27 \%$ & +- \\
\hline $\mathrm{I} 0$ & Alonso & 5 & $23 \%$ & + \\
\hline $\mathrm{II}$ & Felipe & 5 & $23 \%$ & +- \\
\hline $\mathrm{I} 2$ & Kevin & 5 & $23 \%$ & - \\
\hline $\mathrm{I} 3$ & Michael & 5 & $23 \%$ & - \\
\hline $\mathrm{I} 4$ & Pablo & 5 & $23 \%$ & +- \\
\hline $\mathrm{I} 5$ & Alejandro & 4 & $\mathrm{I} \%$ & +- \\
\hline $\mathrm{I} 6$ & Claudio & 4 & $\mathrm{I} \% \%$ & +- \\
\hline $\mathrm{I} 7$ & Jason & 4 & $\mathrm{I} \% \%$ & - \\
\hline $\mathrm{I} 8$ & Jordan & 4 & $\mathrm{I} \% \%$ & - \\
\hline $\mathrm{I} 9$ & Joaquín & 3 & $\mathrm{I} \% \%$ & + \\
\hline 20 & Agustín & 2 & $9 \%$ & + \\
\hline $2 \mathrm{I}$ & Byron & 2 & $9 \%$ & - \\
\hline & & & & \\
\hline
\end{tabular}




\begin{tabular}{|l|c|c|c|c|}
\hline 22 & Marcelo & 2 & $9 \%$ & +- \\
\hline 23 & Martín & 2 & $9 \%$ & + \\
\hline 24 & Rodrigo & 2 & $9 \%$ & +- \\
\hline 25 & Harry & I & $5 \%$ & - \\
\hline 26 & Jeremy & I & $5 \%$ & - \\
\hline 27 & León & I & $5 \%$ & $+/+-$ \\
\hline 28 & Tomás & I & $5 \%$ & + \\
\hline
\end{tabular}

\section{Observaciones}

En los primeros nombres (Sebastián y Cristóbal) las expectativas se cumplen plenamente. Sin embargo, con un 36\% destaca la aparición del nombre Daniel, pues no se preveía que se lo asociara con popularidad, al mismo nivel de nombres como Cristóbal, Ignacio y Máximo.

Posteriormente, con una votación de un 32\%, destaca la presencia del nombre Brian, pues la expectativa era que no se lo asociaría con popularidad, ni superara a un nombre como Alonso.

Algo similar ocurre con los nombres Kevin y Michael (23\%), los cuales -a diferencia de Alonso, con el cual comparte la misma votación- no se preveían asociados con popularidad.

Por último, destaca el nombre Tomás, el cual sí se preveía asociado con popularidad; sin embargo, según la tabla, solo obtiene un 5\% de las votaciones.

Al establecer una comparación con los resultados obtenidos por Salamanca y Pereira (2013), se observa, nuevamente, una cohesión mayor en las votaciones emitidas por los sujetos con un nivel educacional superior, pues en ese caso los nombres considerados fueron 2I, mientras que en el presente estudio la cifra asciende a 28.

Cabe señalar, además, que en ambos estudios los nombres Sebastián y Cristóbal comparten las primeras posiciones.

Otro aspecto digno de mención es que en ambos estudios ocurre que nombres de expectativa neutra se sitúan dentro de las cuatro primeras posiciones: en el caso de Salamanca y Pereira (2013), el 
nombre Rodrigo se sitúa en la cuarta posición, con un 47,36\% de los votos, mientras que en el presente estudio, el nombre Daniel aparece en la tercera posición con un $36 \%$ de las preferencias.

\subsubsection{En relación con la pregunta “¿Cuáles de estos nombres} relacionas con un nivel social bajo?"

\begin{tabular}{|l|c|c|c|c|}
\hline $\mathrm{N}^{\circ}$ & NOMBRE & $\mathrm{N}$ & PORCENTAJE & EXPECTATIVA \\
\hline $\mathrm{I}$ & Héctor & 9 & $4 \mathrm{I} \%$ & +- \\
\hline 2 & Marcelo & 9 & $4 \mathrm{I} \%$ & +- \\
\hline 3 & Alejandro & 8 & $36 \%$ & +- \\
\hline 4 & Andrés & 8 & $36 \%$ & +- \\
\hline 5 & Daniel & 7 & $32 \%$ & +- \\
\hline 6 & Felipe & 7 & $32 \%$ & +- \\
\hline 7 & Mauricio & 7 & $32 \%$ & +- \\
\hline 8 & Pablo & 7 & $32 \%$ & +- \\
\hline 9 & Claudio & 6 & $27 \%$ & +- \\
\hline $\mathrm{I} 0$ & Justin & 6 & $27 \%$ & - \\
\hline $\mathrm{II}$ & Byron & 5 & $23 \%$ & - \\
\hline $\mathrm{I} 2$ & Jordan & 5 & $23 \%$ & - \\
\hline $\mathrm{I} 3$ & Brian & 4 & $\mathrm{I} \% \%$ & - \\
\hline $\mathrm{I} 4$ & Jason & 4 & $\mathrm{I} \% \%$ & - \\
\hline $\mathrm{I} 5$ & Rodrigo & 4 & $\mathrm{I} \% \%$ & +- \\
\hline $\mathrm{I} 6$ & Sebastián & 4 & $\mathrm{I} \% \%$ & + \\
\hline $\mathrm{I} 7$ & Alonso & 3 & $\mathrm{I} \% \%$ & + \\
\hline $\mathrm{I} 8$ & Cristóbal & 3 & $\mathrm{I} \% \%$ & + \\
\hline $\mathrm{I} 9$ & Harry & 3 & $\mathrm{I} \% \%$ & - \\
\hline 20 & Jeremy & 3 & $\mathrm{I} \% \%$ & - \\
\hline $2 \mathrm{I}$ & Kevin & 3 & $\mathrm{I} \% \%$ & - \\
\hline 22 & Malcom & 3 & $\mathrm{I} \% \%$ & - \\
\hline 23 & Martín & 3 & $\mathrm{I} \% \%$ & + \\
\hline 24 & Michael & 3 & $\mathrm{I} \% \%$ & - \\
\hline 25 & Joaquín & 2 & $9 \%$ & + \\
\hline 26 & León & 2 & $9 \%$ & $+/+-$ \\
\hline 27 & Agustín & $\mathrm{I}$ & $5 \%$ & + \\
\hline 28 & Ignacio & $\mathrm{I}$ & $5 \%$ & + \\
\hline & & & & \\
\hline & & & + \\
\hline & 3 & & + \\
\hline & 3 & & + \\
\hline
\end{tabular}




\section{Observaciones}

Llama la atención inmediatamente la aparición de los nombres Héctor y Marcelo en la primera posición, pues no se tenía como expectativa que se asociaran con los estratos bajos. Esta ruptura de las expectativas se mantiene hasta el nombre $\mathrm{N}^{\circ}$ 9, inclusive, puesto que hasta ahí todos se habían previsto con una valoración neutra.

Sorprende, también, la aparición de los nombres Rodrigo, Sebastián, Alonso y Cristóbal (números I5 al I8), pues, con la sola excepción del primero, todos se habían previsto asociados con un estrato alto.

Al establecer una comparación con los resultados obtenidos por Salamanca y Pereira (2013), nuevamente existe una cohesión mucho mayor en las votaciones emitidas por los sujetos con un nivel educacional superior, pues en ese caso los nombres considerados fueron sólo I5, mientras que en el presente estudio la cifra asciende a 28.

Otra diferencia importante reside en que en el trabajo de Salamanca y Pereira (2013) las votaciones de los sujetos cumplen con las expectativas dado que sitúan en las diez primeras posiciones a los I0 nombres con expectativa de valoración negativa. En el presente estudio, en cambio, no se obtienen los resultados expectables, pues en las nueve primeras posiciones aparecen nombres con expectativa de valoración neutra.

Finalmente, es destacable que en el estudio realizado con sujetos de nivel educacional superior, el nombre Byron alcanza un 100\% de menciones en esta pregunta, mientras que en el presente estudio obtiene apenas un $23 \%$.

\subsubsection{En relación con la pregunta “¿Cuáles de estos nombres relacionas con un nivel social alto?"}

\begin{tabular}{|l|c|c|c|c|}
\hline $\mathrm{N}^{\circ}$ & NOMBRE & $\mathrm{N}$ & PORCENTAJE & EXPECTATIVA \\
\hline $\mathrm{I}$ & Máximo & 9 & $4 \mathrm{I} \%$ & $+/+-$ \\
\hline 2 & Brian & 8 & $36 \%$ & - \\
\hline 3 & Felipe & 8 & $36 \%$ & +- \\
\hline 4 & Cristóbal & 7 & $32 \%$ & + \\
\hline
\end{tabular}




\begin{tabular}{|c|c|c|c|c|}
\hline 5 & Ignacio & 7 & $32 \%$ & + \\
\hline 6 & Jeremy & 7 & $32 \%$ & - \\
\hline 7 & Joaquín & 7 & $32 \%$ & + \\
\hline 8 & Sebastián & 7 & $32 \%$ & + \\
\hline 9 & Agustín & 6 & $27 \%$ & + \\
\hline 10 & Alonso & 6 & $27 \%$ & + \\
\hline II & Michael & 6 & $27 \%$ & - \\
\hline 12 & Harry & 5 & $23 \%$ & - \\
\hline $\mathrm{I3}$ & Kevin & 5 & $23 \%$ & - \\
\hline $\mathrm{I} 4$ & Malcom & 5 & $23 \%$ & - \\
\hline I5 & Jason & 4 & $18 \%$ & - \\
\hline 16 & Tomás & 4 & $18 \%$ & + \\
\hline 17 & Claudio & 3 & $\mathrm{I} 4 \%$ & +- \\
\hline 18 & Jordan & 3 & $\mathrm{I} 4 \%$ & - \\
\hline I9 & Justin & 3 & $\mathrm{I} 4 \%$ & - \\
\hline 20 & Martín & 3 & $\mathrm{I} 4 \%$ & + \\
\hline $2 \mathrm{I}$ & Rodrigo & 3 & $\mathrm{I} 4 \%$ & +- \\
\hline 22 & Alejandro & 2 & $9 \%$ & +- \\
\hline 23 & Andrés & 2 & $9 \%$ & +- \\
\hline 24 & Byron & 2 & $9 \%$ & - \\
\hline 25 & Daniel & 2 & $9 \%$ & +- \\
\hline 26 & Héctor & 2 & $9 \%$ & +- \\
\hline 27 & León & 2 & $9 \%$ & $+/+-$ \\
\hline 28 & Pablo & 2 & $9 \%$ & +- \\
\hline 29 & Marcelo & I & $5 \%$ & + \\
\hline 30 & Mauricio & I & $5 \%$ & + \\
\hline
\end{tabular}

\section{Observaciones}

Como se observa, en el primer lugar aparece el nombre Máximo, lo que destaca, pues la expectativa respecto de él fluctuaba entre una asociación con un nivel alto o sin asociación con algún nivel socioeconómico. Con un 36\% destaca el nombre Brian, pues se contrapone claramente a lo previsto. Algo similar ocurre con el nombre Jeremy, puesto que, contra lo expectable, es asignado al estrato alto por un $32 \%$ de los encuestados. 
Luego, destaca el nombre Michael, pues recibe el mismo porcentaje (27\%) que los nombres Agustín y Alonso, respecto de los cuales sí se tenía como expectativa su asociación con el estrato alto.

Una última observación incluye a los nombres Harry, Kevin y Malcom, los cuales sorprendentemente obtienen un $23 \%$ de vinculación con un nivel social alto, superando incluso a los nombres Tomás y Martín.

Al establecer una comparación con los resultados obtenidos por Salamanca y Pereira (2013), nuevamente, se observa una mayor cohesión en las votaciones emitidas por los sujetos con un nivel educacional superior, pues en ese caso los nombres votados fueron 22 , mientras que en nuestro estudio la totalidad de los nombres de la encuesta recibieron alguna mención.

Otro aspecto a destacar es que en el caso de los sujetos con nivel educacional superior la expectativa se cumple en las primeras cinco posiciones; en la presente investigación, en cambio, esta se rompe ya en la segunda posición, ocupada por el nombre Brian, que obtiene el $36 \%$ de las votaciones.

\section{Conclusiones}

En primer lugar, cabe señalar que los objetivos propuestos se cumplieron en la medida en que se determinó el grado de prestigio/ estigmatización que 44 sujetos con vulnerabilidad social asignan a 60 nombres propios, a partir del instrumento utilizado. En efecto, se logró establecer el grado de popularidad/desprestigio que los individuos de la muestra les atribuyen a los nombres estudiados y se estableció la asociación de estos con un estrato sociocultural alto o bajo.

En términos más específicos, se puede señalar que la ocurrencia de una concentración de las preferencias, especialmente, en las preguntas asociadas con estigmatización, habla de una apropiación de los códigos sociales vinculados con prestigio/discriminación por parte de los sujetos, aunque no en un nivel prominente.

Por otra parte, es posible constatar que las votaciones de las mujeres son las que muestran tendencias más sistemáticas. Esto resulta interesante, porque corrobora lo propuesto por investigadores como 
Tassara (1992), quien sostiene que los jóvenes y las mujeres son dos grupos particularmente drásticos al momento de emitir juicios de valor sobre el uso de la lengua.

Otro aspecto destacable es que, salvo una excepción, fue recurrente el hecho de que la cantidad de nombres mencionados por los encuestados/as fue inferior en las preguntas relativas a la estigmatización (nombres con los que serían objeto de burlas y aquellos asociados al nivel bajo); es decir, existe una mayor cohesión en ese tipo de respuestas. Una posible explicación para ello es que si bien los gustos son un aspecto personal, en el momento de calificar un nombre como negativo se manifiesta una mayor consciencia de lo que es socialmente rechazado.

Al comparar, en general, los resultados que se plasman en el artículo de Salamanca y Pereira (2013) con los de la presente investigación, se observa que existe una mayor cohesión en las votaciones emitidas por los sujetos de nivel educacional superior, de ambos sexos.

Uno de los casos sobresalientes en los resultados de los sujetos de género femenino ocurre en la pregunta relativa a los nombres que pueden provocar burla, donde coincide que Shirley se sitúa en la primera posición con un $95 \%$ de los votos. En esta misma línea, pero respecto de los nombres vinculados con un estrato social bajo, se produce, también, una coincidencia, pues este mismo nombre encabeza ambas tablas, pero con una diferencia de porcentajes. En efecto, Shirley recibe un $90,47 \%$ de menciones por parte de los sujetos con nivel educacional superior y solo un $64 \%$ por parte de los sujetos con vulnerabilidad social.

En cuanto a las diferencias más sobresalientes entre ambos estudios, en el caso de nombres asociados con popularidad, destaca el nombre Agustina, el cual obtiene un 66,66\% de los votos de los sujetos con nivel educacional superior, y solo un I4\% por parte de los sujetos con vulnerabilidad social. En los nombres vinculados con un estrato social alto, también, ocurre el fenómeno descrito, pues Agustina recibe un $90,47 \%$ de los votos por parte de los sujetos con nivel educacional superior, y solo un I8\% por parte de los sujetos en situación de vulnerabilidad social. 
En el caso de los resultados arrojados por las encuestas aplicadas a sujetos masculinos, en el ítem referido a los nombres que pueden provocar burla, destaca Brian, que recibe un $84,25 \%$ de las menciones por parte de los sujetos con nivel educacional superior y solamente un $14 \%$ en el presente estudio.

Por otra parte, es importante mencionar el caso de los nombres Sebastián y Cristóbal, los cuales en ambos estudios encabezan las tablas relativas a los nombres con los que serían más populares, cumpliendo así la expectativa de valoración positiva.

Para finalizar, en la pregunta relativa a la asociación con un nivel social alto, conviene mencionar el caso del nombre Agustín, el cual recibe un $52,6 \%$ de los votos por parte de sujetos con nivel educacional superior, ubicándose en el primer lugar de la tabla, mientras que los sujetos con vulnerabilidad social le otorgan un $27 \%$ de menciones, ubicándolo en la novena posición.

Finalmente, como posible proyección se propone realizar un estudio similar en otros países hispanohablantes, donde, tal vez, sean otros nombres los considerados prestigiosos y estigmatizados por la sociedad. Sin duda, los mismos datos ya obtenidos pueden ser objeto de un abordaje estadístico más sofisticado, por medio del empleo de estadígrafos ad hoc, lo que, por cierto, podría iluminar algunos aspectos no tratados aquí o tratados en forma general.

\section{Referencias bibliográficas}

Carbajal, V. (2006). Cambio y conservación intergeneracional del quecbua. La Paz: Plural Editores.

Cooper, R. (1989). La planificación lingüística y el cambio social. Cambridge: Cambridge University Press.

Fasold, R. (1996). La Sociolingüistica de la sociedad. Introducción a la Sociolingüística. Madrid: Visor Libros.

Figueroa, M., Soto, J. \& Nanculeo, M. (2010). Los alófonos del grupo consonántico /tr/ en el castellano de Chile. Onomázein, 22, II- 42.

Figueroa, M., Salamanca, G. \& Nanculeo, M. (2013). El eje oclusión-fricción en el sistema sociofónico del castellano chileno. Estudios en Fonética Experimental, XXII, 233-273. 
Lastra, Y. (I992). Sociolingüística para bispanoamericanos. México: El Colegio de México.

Millán, M. \& Rodríguez, C. (2012). Prestigio y estigmatización de 30 nombres propios en 44 sujetos de estrato sociocultural bajo. Tesis de Pregrado, Universidad de Concepción. Concepción, Chile.

Pujol, M. (2013). Retos y perspectivas del español como recurso económico: el sector de la enseñanza de la lengua para extranjeros. En Alonso, M. \& Furio Blasco, E. Innovando en el mundo de los negocios: La dimensión económica del español. (pp. I29-I37). Oviedo: Septem.

Salamanca, G. (2010). Apuntes sociolingüísticos sobre la presencia de argentinismos en el léxico del español de Chile. Atenea, 502, I25-I49.

Salamanca, G. \& Valverde, A. (2009). Prestigio y estigmatización en variantes anteriorizadas y posteriorizadas de las vocales del español de Chile. Literatura y Lingüística, 20, I25-I40.

Salamanca, G. \& Pereira, L. (2013). Prestigio y estigmatización de 60 nombres propios en 40 sujetos de nivel educacional superior. Universum, 28(2), 35-57.

Silva-Corvalán, C. (200I). Sociolingüística y pragmática del español. Washington D.C.: Georgetown University Press.

Tassara, G. (1992). Actitudes lingüísticas ante la variación de / ĉ́ . Revista de Lingüística Teórica y aplicada, 30, 263-27I.

Valdivieso, H. (1983). Prestigio y estigmatización: factor determinante en la enseñanza institucionalizada de la lengua materna. Revista de Lingüística Teórica y Aplicada, 21, 137-I42. 